\title{
IMPLEMENTAÇÃO DE METODOLOGIA ÁGIL EM ÁREAS DE OPERAÇÕES: UM ESTUDO DE CASO SOBRE A OUVIDORIA DA IBM (INTERNATIONAL BUSINESS MACHINE)
}

\author{
Davi de Pinho Spilleir ${ }^{1}$ \\ Arllon Souza Viçoso² \\ Melissa Schetini ${ }^{3}$
}

\begin{abstract}
Resumo
As metodologias ágeis têm sido amplamente aplicadas ao longo das últimas duas décadas para o desenvolvimento de softwares, pois apresentam melhores práticas de integração entre empresas e stakeholders, maior espaço e agilidade para aplicação de mudanças, além de promoverem pequenas entregas em diferentes etapas da concepção, o que permite a correção de prototipagens ao longo do processo criativo. A literatura e os estudos, entretanto, ainda são bastante limitados com relação à abrangência dessas metodologias, retratando-as restritas unicamente às realidades de equipes voltadas a desenvolvimento de software. $\mathrm{O}$ objetivo deste artigo é lançar luz a uma aplicação diferente do tema, analisando como foi realizada e quais os resultados da implementação de metodologia ágil em todas as equipes de Ouvidoria de uma empresa multinacional de tecnologia. Para se realizar tal objetivo, é construída uma narrativa de Estudo de Caso, com dados coletados através de entrevistas com a equipe de Ouvidoria, cujos resultados servem como um possível norte para novas escritas da temática em áreas não associadas ao desenvolvimento de software, elencando dificuldades e quais os resultados quantitativos da implementação.
\end{abstract}

Palavras-Chave: Metodologia ágil. Implementação em larga escala. Ouvidoria. Empresa de Tecnologia.

1 Mestre em Sustentabilidade pela Pontifícia Universidade Católica de Campinas, pós-graduado em Gestão pela USP de Piracicaba e bacharel em Comércio Exterior pela PUC-Campinas. (dpinho@br.ibm.com)

2 Bacharel em Ciências da Computação pela Universidade Paulista e MBA em Gestão Estratégica de Negócios pela FIAP. Líder do Centro de Complaint Management para a América Latina, Ombudsman da IBM Brasil e do Banco IBM. (arllons@br.ibm.com)

3 Bacharel em Ciências Social pela Universidade de São Paulo. (melsch@br.ibm.com) 


\begin{abstract}
Agile methodologies have been widely applied over the last two decades for the development of software, as they present better integration practices between companies and stakeholders, greater space and agility to apply changes, in addition to promoting small deliveries at different stages of design, which allows the correction of prototypes throughout the entire creative process. Literature and studies, however, are still very limited in relation to the comprehensiveness of these methodologies, portraying them restricted only to the realities of development teams. The objective of this article is to shed light on a different application of this theme, analyzing how it was accomplished and what the results of the implementation of agile methodology in all Ombudsman team of a multinational technology company. To accomplish this goal, a Case Study narrative is constructed, with data collected through interviews with the Ombudsman team, the results of which serve as a possible north for new writing of the subject in areas not associated with software development, listing difficulties and what were the quantitative results of implementation.
\end{abstract}

Keywords: Agile methodology. Large scale implementation. Ombudsman. Technology enterprise. 


\section{INTRODUÇÃO}

As metodologias ágeis têm imposto substanciais mudanças aos setores de tecnologia da informação, em especial à área de desenvolvimento de software, ao longo das últimas décadas. TURNER e COCHRANE (1993) elucidam alguns pontos tidos como negativos sobre a concepção de projetos de desenvolvimento. Na opinião deles, os objetivos eram por demasiado inflexíveis, com baixa interação entre empresa desenvolvedora e cliente final e tinham como principal ideário de qualidade saber se seriam entregues dentro do prazo e orçamento, pouco importando se os produtos seriam úteis aos clientes e seus usuários, ou mesmo se atenderiam às suas expectativas.

Criadas como respostas às antigas orientações de desenvolvimento de software e condução de projetos, as metodologias ágeis destacam-se por enfatizar um design contínuo de produto, cujo escopo não é enrijecido, mas flexível e com constantes contribuições oferecidas pelos clientes em todas as etapas do processo criativo (SERRADOR; PINTO, 2015).

Parte relevante para a execução dessas metodologias está no fato de que as equipes são normalmente pequenas, não excedendo doze pessoas, e multifuncionais (RIGBY; SUTHERLAND; TAKEUCHI, 2016), além disso, a implementação em grande escala ${ }^{4}$ (e não apenas o funcionamento das equipes maiores) é apresentada como um fator dificultador para o sucesso, uma vez que com frequência requer a mudança de toda a cultura organizacional e a necessidade de coordenação interequipes (MISRA et al., 2010; DIKERT, 2016; LINDSJORN et al., 2016).

Notória também é a quantidade de questões sobre a viabilidade, ou não, das metodologias ágeis em áreas que não sejam relativas à tecnologia da informação, como operações, por exemplo. COLTMAN et al. (2015) salientam o risco da aplicabilidade em áreas outras, alegando que pode haver um desnível em questões de entrosamento e maturidade entre as áreas tecnológicas da empresa e de operações que poderia acarretar a latência para se responder às mudanças requeridas. ROSES, WINDMÖLLER e CARMO (2016) e MITTAL (2014) são um pouco mais contundentes e afirmam que as metodologias ágeis não devem ser aplicadas em operações com repetibilidade e/ou em processos demasiadamente burocráticos.

De fato, tal parece ser a aceitabilidade dessas afirmações que não há nenhum artigo nas bases de dados do SciELO e do Google Scholar que tenha lançado qualquer olhar diferente do apregoado pela teoria, ou seja, nos campos de desenvolvimento de software ou de gerenciamento de projetos.

Contudo, uma empresa multinacional norte-americana, a IBM, vem, ao longo da última década, conduzindo um plano que parece fugir à regra habitual estipulada na teoria e expandindo a utilização das metodologias ágeis para seus setores de execução e operações, inclusive já vendendo a expertise adquirida como um serviço, através da prestação de consultoria àqueles que quiserem realizar o mesmo tipo de implementação (IBM, 2019).

Tendo em vista a escassez de estudos acerca da temática e a relevância dessa aplicação para servir de base a estudos posteriores, é objetivo deste trabalho realizar um estudo de caso anali-

4 DIKERT et al. (2016) descrevem como larga escala aquelas operações que excedem cinquenta pessoas, ou que envolvam coordenação de mais do que seis times distintos, podendo, inclusive, ser descrito como um processo iniciado em um piloto e expandido aos poucos para as demais áreas. 
sando as dificuldades e resultados da implementação de metodologia ágil em uma de suas áreas de operações, o time global de Complaint Management (terminologia utilizada para indicar o que no Brasil se entende como Ouvidoria).

\section{METODOLOGIAS ÁGEIS E A IMPLEMENTAÇÃO EM LARGA ESCALA}

A rigorosa competição e o mercado em permanente mudança, em que transformações digitais resultam na constante necessidade por inovação e desenvolvimento, não permitem mais a possibilidade de se questionar "se", mas sim - e cada vez mais - o tempo para adequado e "como" se deve fazer algo diferente, até para a própria sobrevivência da organização. Para as empresas, conviver em constante adaptação é latente. Ou se responde às mudanças, ou estão fadadas à obsolescência (PECH et al., 2017).

Nesses termos, é correto dizer que os desafios relativos à gestão de projetos e desenvolvimento de Tecnologia de Informação (TI) se mostram mais agudos e cada vez menos deixam espaço para soluções padronizadas.

A transformação digital empoderou sensivelmente os clientes, de tal modo que o desenvolvimento de produtos passou a ser centrado nas experiências dos usuários, com constantes realizações de testes e prototipagens, com interações diretas com o público, que viabilizam uma entrega mais adequada ao contexto e às pressões do mercado volátil (CASANOVA, 2019).

Foi nesse contexto que, em 2001, lançou-se o Manifesto para Desenvolvimento Ágil de Software, que apregoava princípios básicos que seriam melhores práticas para a condução de projetos dessa natureza:

- Indivíduos e interações mais que processos e ferramentas.

- Software em funcionamento mais que documentação abrangente.

- Colaboração com o cliente mais que negociação de contratos.

- Responder a mudanças mais que seguir um plano. (FOWLER, HIGHSMITH, 2001, s/n)

A ideia por detrás desse manifesto era que se privilegiassem as ações em negrito em detrimento às outras. Em outras palavras, seria dizer que ações práticas seriam muito mais relevantes e de maior agregação de valor do que ações burocráticas. SERRADOR e PINTO (2015) sintetizaram que a perspectiva mais relevante seria a de tratar como dimensões a serem trabalhadas: a) eficiência do projeto, que seria a adequação aos custos, tempo e escopo; e b) o sucesso do stakeholder, que seria satisfazer as expectativas dos interessados no projeto, que são os melhores para avaliar o sucesso de maneira geral.

LINDSJORN et al. (2016) lançam olhar de que há ganho de sinergia e integração entre os times quando aplicadas metodologias ágeis, haja vista que as tarefas são selecionadas de acordo com seu grau de relevância dentro de uma cerimônia de planejamento, havendo a possibilidade de serem divididas em pequenas ações e posteriormente ou delegadas ou escolhidas pelos membros. TJOSVOLD (1998) ressalta que os membros devem ajudar-se mutuamente através de constantes 
feedbacks e levantando dúvidas ou necessidades em reuniões diárias para avaliar o progresso do que foi proposto contra o que foi feito. Na concepção de MÜLLER e COPPER (1994), isso resulta em três aspectos de coesão da equipe: i) engajamento para com as tarefas; ii) atração interpessoal; e iii) orgulho e sentido de pertencimento de equipe.

RIGBY, SUTHERLAND e TAKEUCHI (2016) demonstram em seus estudos que os benefícios marginais dessas práticas não se restringem apenas a entregas mais personalizadas aos clientes ou maior sinergia entre as equipes, mas também no fato de que implementações de projetos em metodologias ágeis tendem a ser até $60 \%$ mais bem-sucedidas, além de gerarem melhor sensação de valor nas soluções recebidas.

Contudo, ao se falar em metodologias ágeis, apesar de representarem uma mudança de organização e priorização do trabalho, elas são principalmente uma mudança de mentalidade. Talvez por isso autores sejam tão unânimes em frisar a dificuldade em implementá-las em larga escala.

DIKERT, PAASIVAARA e LASSENIUS (2016) descrevem que o principal empecilho para o bom resultado de larga escala é a necessidade de coordenação adicional interequipes. NERUR et al. (2005) ressaltam que há três óticas essenciais para serem levadas em consideração sobre as equipes: o impacto da cultura do indivíduo, do grupo e da organização na aplicação das metodologias. COLTMAN et al. (2015) discorrem que implementações demasiadamente grandes tendem a perder a capacidade de responder às mudanças ambientais com rapidez.

FREUNDENBERG e SHARP (2010) demonstram que, na ocasião da conferência XP2010, as principais questões dos executivos envolvidos concerniam à viabilidade - ou não - de implementações de metodologias ágeis em larga escala. MOE e DINGSOYR (2017), ao analisarem a quinta versão do exemplo, percebem que essas dúvidas persistiam ainda após uma década, porém se notava que alguns casos de sucesso, nas áreas de desenvolvimento e projetos, haviam sido reportados.

Experiência interessante e de grande valor acadêmico é a implementação de metodologia ágil levada a cabo pela IBM, empresa do setor tecnológico e de serviços, que não se restringiu a fazê-la unicamente para as áreas técnicas, mas também para suas equipes de operações. Tendo em vista a singularidade e a relevância do objeto de apreço, o escopo de trabalho restringe-se a retratar a viabilização na área de Complaint Management (Ouvidoria).

\section{PROCEDIMENTOS METODOLÓGICOS}

A fim de operacionalizar o presente trabalho, contar-se-á com uma metodologia qualitativa, que, nas palavras de GODOY (1995), se embasa na possibilidade de estudar fenômenos que envolvem os seres humanos e suas intrincadas relações sociais, estabelecidas em diversos ambientes e com a intenção de explicar o específico.

Assim, optou-se pela realização de um estudo de caso, o que, segundo YIN (2001), sagra-se como sendo uma investigação empírica de um fenômeno contemporâneo dentro de um contexto da vida real, nesse caso, a implementação da metodologia ágil (doravante chamada de agile) na empresa IBM. 
A seleção da IBM para ser objeto de estudo dá-se por ser uma das seis maiores empresas de tecnologia do mundo (FORBES, 2019), que, centenária, ainda assim consegue manter-se à frente das tendências, investindo em áreas de fomento e desenvolvimento de novas tecnologias (KNEBEL, 2018). Ademais, seu projeto de implantação de agile em suas áreas de operações, mais notadamente em um time global de Ouvidoria, é uma novidade teórica, e portanto de valia para embasar trabalhos exploratórios futuros.

Para a obtenção de material, tomaram-se dois rumos consonantes de pesquisa: de um primeiro ponto, houve uma coleta de dados não padronizada realizada com o líder da área de negócios e Ombudsman da empresa para que se entendesse como se deu a condução, alguns pontos notáveis, datas e, enfim, se tivesse a percepção clara do ponto de vista gerencial do que foi realizado.

Em um segundo momento, a coleta de dados foi dirigida à equipe de Ouvidoria. Mais uma vez aplicou-se uma entrevista não padronizada para se obter o ponto de vista deles, especialmente no tocante às operações, como cerimônias que passaram a ser realizadas e o entendimento do modus operandi da equipe.

A aplicação de ambos instrumentos de coleta de dados foi aprovada pela linha executiva pertinente às operações de Ouvidoria, e os membros da equipe (os entrevistados) devidamente informados sobre o procedimento que estava sendo feito.

Ao final foram disponibilizadas as métricas do período de 2018 e 2019, para que se pudesse traçar históricos, verificar tendências e realizar uma análise qualitativa e tratamento desses dados. Para a análise dos materiais será usada a estratégia do explanation building, ou construção da explanação. YIN (2001) discorre que, nessa estratégia, tem-se como objetivo a construção de um repertório analítico de maneira narrativa, justapondo e enfatizando aquilo importante no confronto dos elementos teóricos com os achados da pesquisa.

\section{RESULTADOS E DISCUSSÃO}

A equipe de Ouvidoria da IBM possui abrangência global, dividindo-se em pequenas equipes, situadas em diferentes partes do globo, cujas ações são divididas especificamente pela proximidade geográfica. As equipes dividem-se da seguinte maneira: América Latina, situada em SP; América do Norte, situada em várias cidades dos Estados Unidos; China e Coreia, em Dalian; Ásia e Pacífico, em Brisbane; Europa, Mediterrâneo, Leste Europeu e África, em Bratislava.

Desses times, apenas o da América do Norte não fez parte da implementação da metodologia ágil (agile) nas operações de Ouvidoria. A execução começou como um piloto independente, em 2016, na região da América Latina, contudo foi conduzida de maneira oficial em todas as geografias em 2018, através de um programa denominado Accelerate, que realizou a implementação em cada uma dessas geografias escalonadamente, com entrega definitiva em fevereiro de 2019 e com a participação de aproximadamente sessenta pessoas em todo o mundo.

A ideia por detrás do programa era realmente realizar uma mudança na cultura das equipes de Ouvidoria, modificações que gerassem sensíveis melhoras na percepção do valor recebido 
pelos clientes, e para isso foi feita uma série de reestruturações, como a redução de camadas hierárquicas, modificação nas métricas a fim de se identificar gargalos e as entregas dos chamados de Ouvidoria sendo muitas vezes parceladas em pequenas ações, priorizadas e discutidas em iterações de duas semanas.

Talvez a implementação parcelada, isto é, em cada uma das geografias separadamente, tenha reduzido sensivelmente os problemas que seriam advindos da implementação em larga escala, pois cada uma das equipes teve a possibilidade de moldar a metodologia de acordo com suas nuances, sem perder a característica principal das cerimônias e do modus operandi. Atualmente, trabalha-se no último ponto a se fechar, que é a padronização em nível global de todas os processos executados pelas equipes de Ouvidoria.

Sobre a remodulagem das equipes, com a redução das camadas de hierarquia, a equipe tem o desafio e a oportunidade de exercer papéis de maior liderança, já que a função de Iteration Manager não é atribuída a uma pessoa específica, mas é temporária e rotativa. Portanto todos os membros da equipe em algum momento são responsáveis por facilitar as cerimônias ágeis do grupo, buscar e apresentar as métricas, liderar reuniões e os encontros diários, entre as demais atribuições da posição. Os funcionários de todos os níveis e/ou senioridade têm a mesma oportunidade e responsabilidade de se colocar numa posição de influência dentro da equipe, o que representa tanto um desafio individual para cada um deles quanto um espaço para desenvolvimento profissional e pessoal, acessível a todos igualmente.

Após o momento inicial em que os funcionários mais experientes se revezavam no exercício dessa função, todos os outros também passaram a se sentir confortáveis para se colocar como Iteration Managers por determinados períodos.

Com a adoção da metodologia agile, a equipe passou a participar mais ativamente das decisões que afetam a todo o grupo, em definições que anteriormente eram vistas como responsabilidade apenas gerencial. O grupo participa inclusive das entrevistas de novos funcionários a serem contratados, quando tem a possibilidade de fazer perguntas, avaliar currículos e ter suas opiniões sendo consideradas dentro desses processos de seleção.

Os objetivos a serem alcançados em cada iteração são definidos e priorizados em conjunto, levando-se em conta as expectativas de clientes e stakeholders. Há, portanto, clareza e transparência em relação aos resultados esperados, que são acompanhados e medidos pelo próprio grupo ao final de cada período, quando eles próprios podem analisar seu desempenho e, juntos, avaliar casos de sucesso e definir planos de ação para os pontos que precisam de correção, desenvolvimento ou melhoria.

Os principais indicativos usados para avaliação de desempenho e performance são o Cycle Time (tempo de solução de um registro de uma demanda) e o Net Promoter Score (NPS), que é uma avaliação do cliente interno ou externo em relação à sua satisfação com a companhia e com o trabalho da Ouvidoria, numa escala de 0 a 10.

Hoje, passada a fase de implementação do agile, já é possível verificar a condição dos resultados na comparação entre os anos de 2018 e 2019. Uma vez que o volume de casos da Ouvidoria tem grande variação ao longo do ano, utilizou-se como base de comparação os primeiros cinco meses do ano de 2018, janeiro a maio (antes da implementação), e os primeiros cinco meses de 
2019 (pós-implementação), para analisar se, apesar do pouco tempo de prática da metodologia ágil, já seria possível encontrar algum impacto nesses resultados.

Em relação ao Cycle Time, houve uma melhora de 8,9\% na comparação entre os primeiros meses de 2018 e o mesmo período de 2019. Quanto às pesquisas de satisfação, os resultados meIhoraram 10,5\% em relação às pesquisas com clientes externos e 13,5\% com os clientes internos.

Além das métricas de desempenho, com a implementação do agile, equipe e gerência também realizam o acompanhamento da satisfação dos funcionários, obtido através de uma pesquisa diária respondida espontânea e anonimamente, conhecida no meio ágil como Mood Marbles. Os feedbacks e resultados dessa pesquisa são trabalhados por todos, de forma a buscar sempre a manutenção e melhora do clima e da satisfação dos funcionários.

Por fim, com relação às três mais sensíveis vertentes de impacto na aplicação das metodologias ágeis (impacto da cultura do indivíduo, do grupo e da organização), foi possível notar diferenças substanciais, em especial no que tange ao comportamento em grupo. Há um senso de responsabilização coletiva bem mais aflorado, em que os indivíduos reconhecem maior sentido de pertencimento e valor agregado às tarefas agregadas.

\section{CONSIDERAÇÕES FINAIS}

Através do exposto, é possível notar que o trabalho atinge seu objetivo inicial de explorar as nuances da implementação da metodologia ágil nas operações de Ouvidoria no nível global da IBM.

Das diferenças substanciais mais notadas, é possível destacar a relação dos indivíduos para com a mentalidade da nova maneira de trabalhar e a maneira de priorizar as tarefas a serem executadas, com decisões tomadas conjuntamente sobre o que fazer nas semanas sucessivas. A despeito da curta série temporal para análise, já é possível perceber reduções no tempo de resolução dos casos e aumento nas notas dadas pelos clientes para a resolução dos casos, quando comparados os dois períodos destacados no artigo (primeiro semestre de 2018 e primeiro semestre de 2019).

O presente estudo também traz a significativa contribuição de ser uma implementação de metodologias ágil em larga escala (com aproximadamente sessenta pessoas), localizadas em todos os continentes, com padronização no modo de operação. Nota-se que efetivamente a dificuldade desse projeto aumenta proporcionalmente à medida que sejam envolvidas mais pessoas no processo, contudo, apesar de dificultador, não se mostrou um assunto paralisante na análise em questão.

Recomenda-se, para trabalhos futuros, que os olhares se foquem em pontos não cobertos por esse estudo ou com maiores abrangências temporais, que permitam traçar tendências com melhor precisão. Além de relatar dificuldades advindas da implementação global, muito mais do que as etapas que deram certo, pois elas podem contribuir ativamente para a aplicação em demais lugares.

\section{REFERÊNCIAS}

CASANOVA, N. C. Identificação dos desafios inerentes à aplicação de métodos ágeis em grandes organizações. Dissertação. FGV. Orient. MAIA, M. C. 2019, Disponível em: <https://bibliotecadigital.fgv.br/dspace/bitstream/hand- 
le/10438/27575/VF.ProtocoloFinal_NikolasCasanova_MPGCTI.pdf?sequence=1\&isAllowed=y> Acesso em: 25 jun. 2019.

COLTMAN, T.; TALLON, P.; SHARMA, R.; QUEIROZ, M. Strategic IT alignment: twenty-five years on. Journal of Information Technology, v. 30, p. 91-100, 2015. Disponível em: <https://journals.sagepub.com/doi/abs/10.1057/ jit.2014.35> Acesso em: 24 jun. 2019.

DIKERT, K.; PAASIVAARA, M.; LASSENIUS, C. Challenges and success factors for large-scale agile transformations: A systematic literature review. The Journal of Systems and Software, v. 119, p. 87-106, 2016. Disponível em: <https://www.sciencedirect.com/science/article/pii/S0164121216300826> Acesso em: 25 jun. 2019.

FORBES. Global 2000: as maiores da tecnologia em 2019. Disponível em: <https://forbes.uol.com.br/listas/2019/05/ global-2000-os-maiores-da-tecnologia-em-2019/\#foto6> Acesso em: 26 jun. 2019.

FOWLER, M.; HIGHSMITH, J. Manifesto para Desenvolvimento Ágil de Software. 2001. Disponível em: <http://agilemanifesto.org/iso/ptbr/manifesto.html> Acesso em: 25 jun. 2019.

FREUDENBERG, S.; SHARP, H. The Top 10 Burning Research Questions from Practitioners. IEEE, v. 27, n. 5, p. 8-9, 2010. Disponível em: <https://ieeexplore.ieee.org/document/5551011> Acesso em: 24 jun. 2019.

GODOY, A. S. Pesquisas qualitativas, tipos fundamentais. Revista de Administração de Empresas, v. 35, n. 3, p. 2029, 1995. Disponível em: <http://www.scielo.br/pdf/rae/v35n3/a04v35n3.pdf> Acesso em: 26 jun. 2019.

IBM. Serviços ágeis de consultoria de negócios e transformação. Disponível em: <https://www.ibm.com/br-pt/services/business/agile> Acesso em: 25 jun. 2019.

KNEBEL, P. IBM reforça estratégia junto a ecossistemas de inovação. Jornal do Comércio. Disponível em: <https:// www.jornaldocomercio.com/_conteudo/economia/2018/10/651798-ibm-reforca-estrategia-junto-a-ecossistemas-de-inovacao.html> Acesso em: $\overline{26}$ jun. 2019.

LINDSJORN, Y. et al. Teamwork quality and project success in software development: A survey of agile development teams. The Journal of Systems and Software, v. 122, p. 274-286, 2016. Disponível em: <https://www.sciencedirect. com/science/article/pii/S016412121630187X> Acesso em: 25 jun. 2019.

LIVERMORE, J. A. Factors that significantly impact the implementation of an agile software development methodology. Journal of Software, v. 3, n. 4, p. 31-36, 2008. Disponível em: <http://www.jsoftware.us/vol3/jsw0304-04.pdf> Acesso em: 24 jun. 2019.

LU, Y.; RAMAMURTHY, K. Understanding the link between information technology capability and organizational agility: An empirical examination. Mis Quarterly, p. 931-954, 2011. Disponível em: <https://pdfs.semanticscholar.org/e741/ b9e3e0e942c1bd16860b8e2ab693a14d4210.pdf> Acesso em: 25 jun. 2019.

MITTAL, P. Agile: when to use and when NOT to! Disponível em: <https://pt.coursera.org/lecture/agile-software-development/agile-when-to-use-and-when-not-to-5MxQG> Acesso em: 25 jun. 2019.

MOE, N. B.; DINGSOYR, T. Emerging Research Themes and updated Research Agenda for large-Scale Agile Development: A Summary of the 5th International Workshop at XP2017. Disponível em: <https://dl.acm.org/citation. cfm?id=3120474> Acesso em: 25 jun. 2019.

MULLEN, B.; CAROLYN, C. The relation between group cohesiveness and performance: An integration. Psychological Bulletin, v. 115, n. 2, p. 210-227, 1994. Disponível em: <https://psycnet.apa.org/doiLanding?doi=10.1037\% 2F0033-2909.115.2.210> Acesso em: 24 jun. 2019.

NERUR, S.; MAHAPATRA, R.; MANGALARAJ, G. Challenges of migrating to agile Methodologies. Communications of the ACM, v. 48, n. 5, p. 72-78, 2005. Disponível em: <https://dl.acm.org/citation.cfm?id=1060712> Acesso em: 25 jun. 2019.

PECH, J.; PAGLIARINI, D.; NORO, D. C.; VIANA, A. T. A influência da tecnologia no desenvolvimento organizacional. Revista Maiêutica Indaial, v. 5, n. 1, p. 127-136, 2017. Disponível em: <https://publicacao.uniasselvi.com.br/index. php/GESTAO_EaD/article/.../1721/833> Acesso em: 26 jun. 2019.

RIGBY, D. K.; SUTHERLAND, J.; TAKEUCHI, H. Embracing Agile. Harvard Business Review, v. 5, p. 40-48, 2016. Disponível em: <https://hbr.org/2016/05/embracing-agile> Acesso em: 24 jun. 2019.

ROSES, L. K.; WINDMÖLLER, A.; CARMO, E. A. Favorabilidade na adoção de práticas de métodos ágeis no desenvolvimento de software em um banco público. Journal of Information Systems and Technology Management, São Paulo, v. 13, n. 3, set.-dez. 2016. 
SERRADOR, P.; PINTO, J. K. Does agile work? - a quantitative analysis of agile project success. International Journal of Project Management, v. 33, p. 1040-1051, 2015. Disponível em: <https://people.eecs.ku.edu/ hossein/811/ Papers/Agility/does-agile-work.pdf> Acesso em: 24 jun. 2019.

TURNER, J. R.; COCHRANE, R. A. Goals and methods matrix: coping with projects and with ill-defined goals and/ or methods achieving them. International Journal of Project Management, v. 11, n. 2, p. 93-101, 1993. Disponível em: <https://www.sciencedirect.com/science/article/abs/pii/026378639390017H> Acesso em: 24 jun. 2019.

YIN, R. K. Estudo de caso: planejamento e métodos. Tradução de Daniel Grassi. 2. ed. Porto Alegre: Bookman, 2001. 